\title{
Enhancement of the Achievable throughput in Multi-Taper MIMO Spectrum Sensing using Cooperative Hard Decision Fusion Rules
}

\author{
Mohamed Ismail \\ Electronics \& Communications Department \\ Modern Academy for Engineering and Technology \\ Cairo, Egypt
}

\author{
Atef Ghuniem \\ Department of Electrical Engineering \\ Suez Canal University \\ Ismailia, Egypt
}

\author{
Abdelhamid Gaafar \\ Electronics \& Communications Department \\ ASST- Arab Academy for Science, \\ Technology \& Maritime Transport \\ Cairo, Egypt
}

\begin{abstract}
Cognitive radio is known as a smart technology due to its ability to adjust the operating parameters, according to the given conditions and environment. In this paper, a cooperative MT-MIMO (Multi-Taper Multiple Input Multiple Output) spectrum sensing system is proposed in order to enhance the sensing performance and the achievable throughput using different number of antennas. The obtained results of MT-MIMO are compared to the obtained results of Periodogram MIMO (PED-MIMO) under two scenarios. The first scenario is considered for single user detection while the second one is considered for multiple user detection. The proposed system should counteract the problem of hidden primary user nodes.
\end{abstract}

Keywords-Multi-Taper MIMO spectrum sensing; Periodogram-MIMO spectrum sensing; Cooperative Spectrum sensing; Achievable Throughput

\section{INTRODUCTION}

Over the last two decades, wireless technologies have grown rapidly and more spectrum resources are necessary to support increasing expanded wireless services. Multi-Taper Spectrum Sensing (MTSS) technique is used to sense the spectrum trying to enhance the achievable throughput criteria in a small sensing time [1].

The licensed spectrum of the primary user is largely underutilized in large temporal areas [2].Cognitive radio technology was recently proposed in order to determine whether the sensed spectrum is free or busy [3]. It can improve the efficient spectrum usage by allowing secondary users to borrow unused spectrum holes from primary network users to share the primary network spectrum.

As a smart wireless communication system, a cognitive radio knows as the radio frequency sensor. It adopts the communication parameters such as bandwidth, frequency, and transmission power to optimize the spectrum utilization and adjusts its transmission and reception operation. Spectrum sensing is the most effective components of cognitive radio. Through sensing and adapting the communication parameters, a cognitive radio has the capability to use the free spectrum holes and serve the secondary users (SU). This had been done without causing interference to the primary users (PU). To decide whether a PU exists or not, The MTM (Multi-taper
Method) spectrum estimate in the frequency domain was compared against the noise variance in the time domain [4].

The hidden terminal problem is one of the greatest challenges of implementing spectrum sensing. It occurs when cognitive radio doesn't detect the presence of PU due to multipath fading or high penetration loss inside buildings, while a primary user (PU) is operating in the neighborhood [5]. By using multiple cognitive radio users that can cooperatively work, the hidden node problem can be decreased. By increasing the number of CR (Cognitive radio) spectrum sensing nodes and work cooperatively, the spectrum sensing performance can be more improved [6]-[11].

In this paper, the optimization of cooperative MT-MIMO spectrum sensing for maximizing the Achievable throughput even in small sensing time and low signal to noise ratio (SNR) is considered.

The rest of the paper is organized as follows; in section II the system model is presented. In section III the effect of MTMIMO on the detection probability and the false alarm probability are presented. Discussion of the numerical results, simulations in the mentioned Cooperative MT-MIMO spectrum sensing and PED-MIMO Cooperative spectrum sensing are presented in Section IV. Finally, in Section V the conclusions are given.

\section{PROPOSED SYSTEM MODEL}

The proposed system model structure as shown in Fig.1.It consists of $N_{t}$ transmitting antennas and $\mathrm{M}_{\mathrm{r}}$ receiving antennas with channel coefficients $h_{i, j}$ where $i=1, \ldots \ldots . M_{r}$ and $j=1, \ldots \ldots \ldots \ldots N_{t}$. The two hypothesis tests are as follows:

$$
\begin{array}{cl}
H_{0}: y_{i}(n)=w_{i}(n) & n=0, \ldots \ldots, N-1 \\
H_{1}: y_{i}(n)=h_{i, j}(n) x_{j}(n)+w_{i}(n) & n=0, \ldots \ldots ., N-1
\end{array}
$$

It can be written in matrix form as:

$$
H_{0}: Y=W
$$




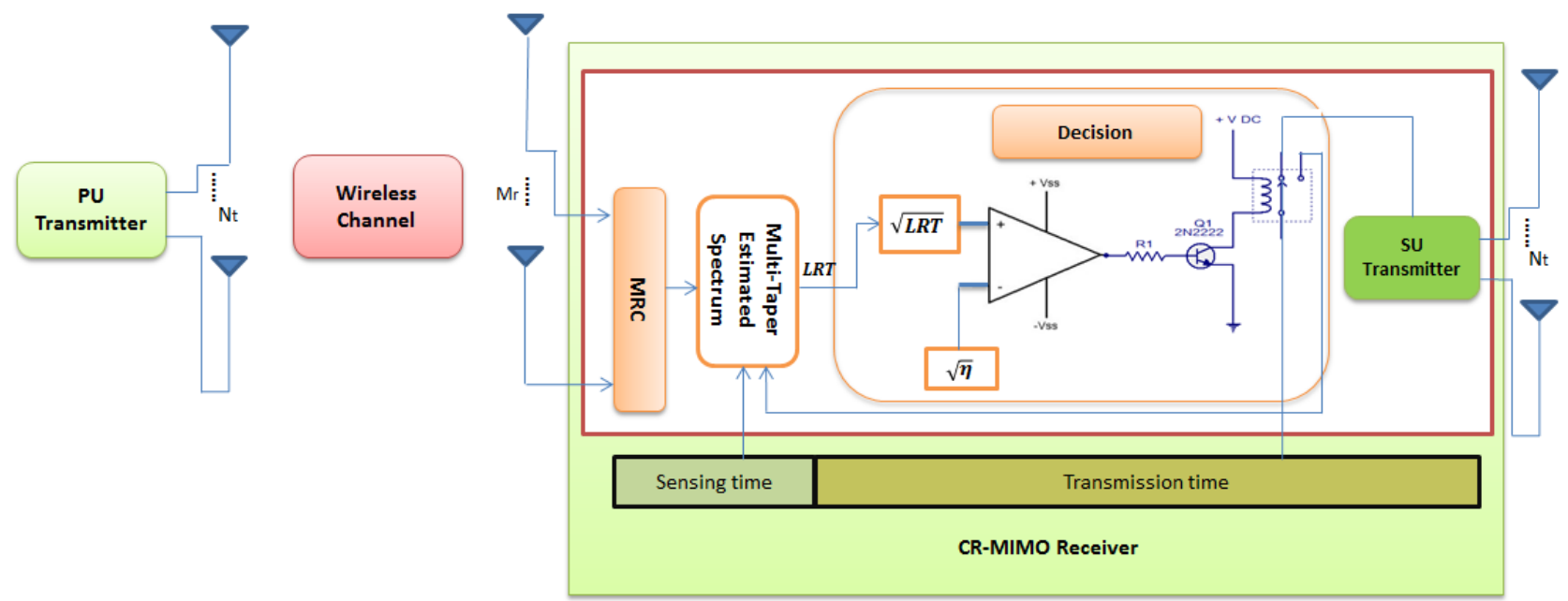

Fig.1The proposed system model structure

where $\mathrm{H}_{0}, \mathrm{H}_{1}$ indicates that the channel is free or busy respectively, $\mathrm{H}$ is the $\mathrm{N}_{\mathrm{t}} \times \mathrm{M}_{\mathrm{r}}$ channel matrix, $\mathrm{N}$ is the number of received sequence and $\mathrm{w}_{\mathrm{i}}($.$) is AWGN associated with \mathrm{i}$ channel weight and assumed to be $N \sim\left(0, \sigma_{t}^{2}\right)$.

To decide whether the observation vector $\mathrm{Y}$ was created under $\mathrm{H}_{0}$ or $\mathrm{H}_{1}$ spectrum sensing is considered. This can be done by formulation appropriate test statistic and comparing it with a predetermined threshold value $\eta$. In the scope of Neyman-Pearson (NP) criterion, the LRT maximization form [11]:

$$
L R T=\ln \left\{\frac{f\left(Y / H_{1}\right)}{f\left(Y / H_{0}\right)}\right\}_{\mathrm{H} 0}^{\mathrm{H} 1} \begin{aligned}
& \mathrm{\sigma} \\
& \mathrm{H}
\end{aligned}
$$

$f($.) is the conditional probability density function (PDF). In logarithmic form

$$
\begin{gathered}
L R T=L_{1}(\mathrm{x})-L_{0}(\mathrm{x}) \\
L_{1}(\mathrm{x})=\frac{-\operatorname{tr}(\mathrm{x})^{2}}{2\left(\sigma_{n}^{2}+\sigma_{s}^{2}\right)}-\frac{L}{2} \ln \left(\sigma_{n}^{2}+\sigma_{s}^{2}\right)-\frac{L}{2} \ln (2 \pi)
\end{gathered}
$$

Where $\operatorname{tr}($.$) is the trace of the matrix.$

$$
\begin{gathered}
L_{0}(\mathrm{x})=\frac{-\operatorname{tr}(\mathrm{x})^{2}}{2 \sigma_{n}^{2}}-\frac{L}{2} \ln \left(\sigma_{n}^{2}\right)-\frac{L}{2} \ln (2 \pi) \\
L R T=-\frac{\operatorname{tr}(\mathrm{x})^{2}}{2\left(\sigma_{n}^{2}+\sigma_{s}^{2}\right)}-\frac{L}{2} \ln \left(\sigma_{n}^{2}+\sigma_{s}^{2}\right)+\frac{\operatorname{tr}(\mathrm{x})^{2}}{2 \sigma_{n}^{2}}+\frac{L}{2} \ln \left(\sigma_{n}^{2}\right) \\
L R T=\frac{\operatorname{tr}(\mathrm{x})^{2}}{2 \sigma_{n}^{2}}\left(1-\frac{1}{1+\frac{\sigma_{s}^{2}}{\sigma_{n}^{2}}}\right)-\frac{L}{2} \ln \left(\frac{\sigma_{n}^{2}+\sigma_{s}^{2}}{\sigma_{n}^{2}}\right)
\end{gathered}
$$

$$
L R T=\frac{\operatorname{tr}(\mathrm{x})^{2}}{2 \sigma_{n}^{2}}\left(\frac{1}{1+\frac{\sigma_{s}^{2}}{\sigma_{n}^{2}}}\right)-\frac{L}{2} \ln \left(1+\frac{\sigma_{s}^{2}}{\sigma_{n}^{2}}\right)
$$

Comparing the LRT function with a threshold results as:

$$
\frac{\operatorname{tr}(\mathrm{x})^{2}}{2 \sigma_{n}^{2}}\left(\frac{1}{1+\frac{\sigma_{s}^{2}}{\sigma_{n}^{2}}}\right)-\frac{L}{2} \ln \left(1+\frac{\sigma_{s}^{2}}{\sigma_{n}^{2}}\right) \stackrel{\mathrm{H} 1}{\mathrm{O}} \eta
$$

Where $\eta$ is the decision threshold and $\sigma_{s}^{2} / \sigma_{n}^{2}$ is the Signal to Noise Ratio (SNR).

\section{MULTI-TAPER MIMO SPECTRUM SENSING}

The multi-taper MIMO method utilizes multi-antenna arrangement environments for spectrum sensing. The Maximal Ratio Combining (MRC) is utilized as near to optimum for diversity combining technique, to approach the estimate spectrum at the output of multiple receiving antennas. The MRC output is the summation of all branches affected appropriately by the used tapers. The classifications of decision statistics differ in the hypothesis test literature and nearly extend either in the time or frequency domains. Each statistical approach is highly relevant to the mathematical models governing signals and systems [13].

The decision proposed earlier was also compared to the noise variance appearing in the time domain. A different extension of the same analysis can also be associated in [14]. In the proposed system model the square root of LRT (Likelihood Ratio Test) of Multi-Taper Estimated Spectrum output is applied to a comparator with reference square root of threshold $\eta$. The comparator output is applied to a transistor through a resistor. The transistor output controls the data transmitted through the transmission time or not. When the 
output of transistor is high, the transistor conducts and the relay is activated so, the data of primary user is transmitted. Otherwise, the same technique is applied to the other output line which referring to sensing again and hence no data is transmitted. Referring to equation (3), this leads to the probability of detection and false alarm probability given as $[15,16$, and 17$]$ :

$$
\begin{gathered}
P_{d}=Q\left(\frac{\eta-L P M_{t} N_{r}(1+\mathrm{SNR})}{\sqrt{2 L P M_{t} N_{r}(1+\mathrm{SNR})}}\right) \\
P_{f}=Q\left(\frac{\eta-L P M_{t} N_{r}}{\sqrt{2 L P M_{t} N_{r}}}\right)
\end{gathered}
$$

Where $\mathrm{L}$ is sequence length and $\mathrm{P}$ is number of tapers $=4, \mathrm{Q}$ (.) is the right-tail probability for normal Gaussian random variables and $Q(u)=1 / 2 \operatorname{erfc}(u / \sqrt{2})$ for any fixed value $u$. It is shown that the estimate spectrum performance in Multi-Taper MIMO environment depends on the number of transmitting and receiving antennas. This will maximize the detection and false alarm probability under low SNR. Now, the tradeoff between sensing capability and achievable throughput of the SU can be studied under different Cooperative spectrum sensing hard decision AND, OR, and Majority rules [1].

$$
\mathrm{R}_{T}(\mathrm{~S})=\frac{T-S}{T}\left[C_{0}\left(1-\mathrm{P}_{f}\right) \mathrm{P}\left(\mathrm{H}_{0}\right)+C_{1}\left(1-\mathrm{P}_{d}\right) \mathrm{P}\left(\mathrm{H}_{1}\right)\right]
$$

Such that $\mathrm{R}_{T}(\mathrm{~S})$ is the achievable throughput, the channel capacities $C_{0}$ and $C_{1}$ are considered the capacities when the PU is absent or present respectively, $P\left(\mathrm{H}_{0}\right)$ is the probability that the channel is idle and $P\left(\mathrm{H}_{1}\right)$ is the probability that the channel is busy and $T, S$ is the transmission time and sensing time respectively [1].

\section{SIMULATION RESULTS}

In this section, the proposed spectrum sensing system model scheme is evaluated. The factors taken are Slepian tapers of 4 taps. The MT-MIMO CR frame time is $\mathrm{T}=40 \mathrm{~ms}$, sampling frequency $0.1 \mathrm{MHz}$ and target probability of detection is set to 0.9 .

Fig. 2 shows the relation between the detection probability against SNR for different number of antennas used in case of PED-MIMO spectrum sensing. To satisfy $\mathrm{Pd}=0.9$ the SNR must be $(-8.5,-10,-11)$ for $(1,2,3)$ antenna(s) used respectively. But in case of MT-MIMO to satisfy $\mathrm{Pd}=0.9$, the SNR must be $(-12,-13.3,-14) \mathrm{dB}$ for $(1,2,3)$ antenna(s) used respectively as shown in Fig.3. This indicates that MT-MIMO combining technique can improve the detection probability at low SNR compared to PED-MIMO combining technique.

Fig.4 shows the relation between the achievable throughput against sensing time in case of PED-2x1MIMO for single user detection compared to cooperative spectrum sensing (AND,OR,MAJORITY) different fusion rule detection. The maximum achievable throughput is $(0.78,1.75,0.13,0.6)$ for (PED-2x1MIMO, AND, OR, MAJORITY) at sensing time (6, $4,18,10)$ respectively. But in case of using MT-2x1 MIMO spectrum sensing, the maximum achievable throughput is $(1.15,2.05,0.6,1.15) \mathrm{b} / \mathrm{s} / \mathrm{Hz}$ at sensing time $(8,4,18,10) \mathrm{msec}$ in case of (MT-2x1MIMO, AND, OR, MAJORITY) respectively as shown in Fig.5.

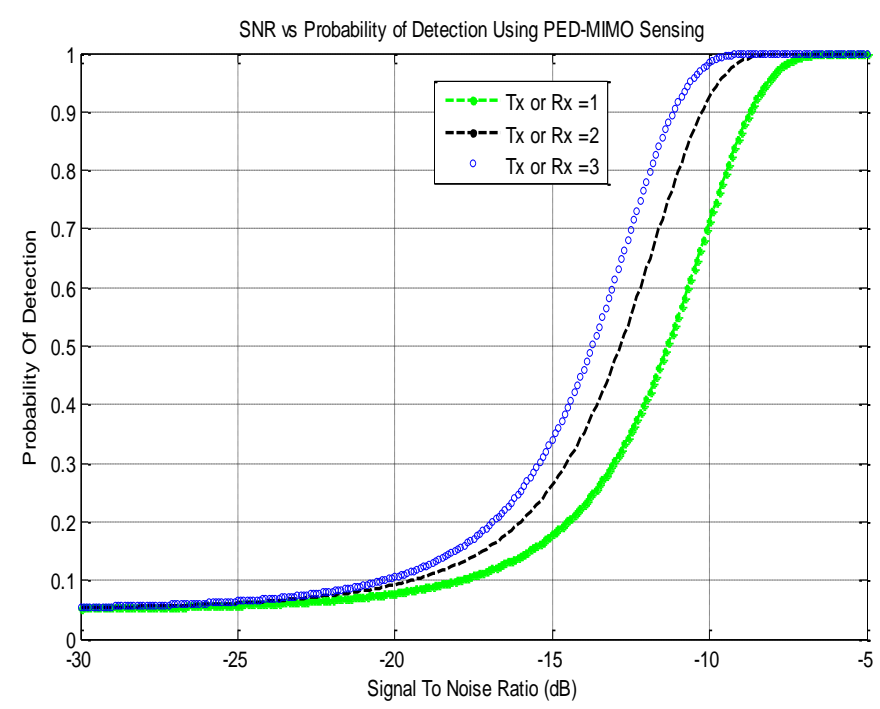

Fig.2. Periodogram-MIMO Pd versus SNR

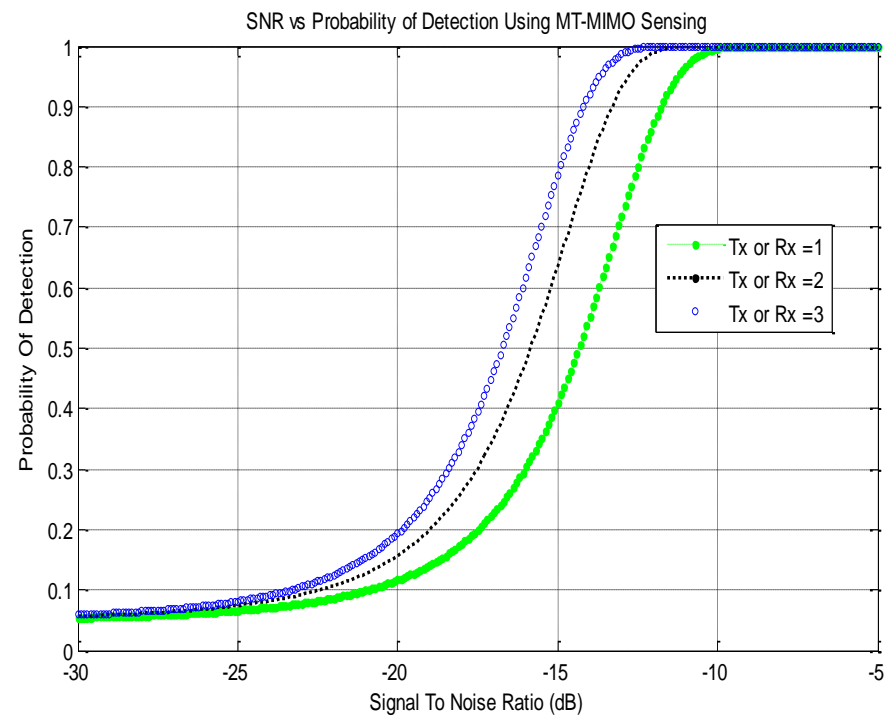

Fig.3 MT-MIMO Pd versus SNR

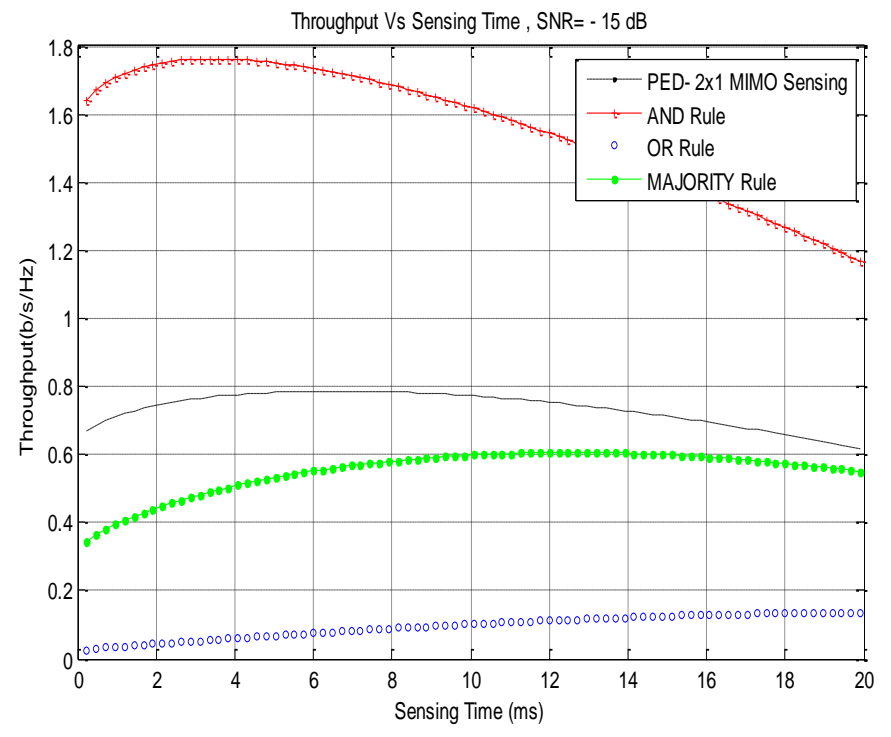

Fig.4 Throughput versus Sensing Time $(\mathrm{SNR}=-15, \mathrm{~K}=3$, PED-2x1 MIMO) 


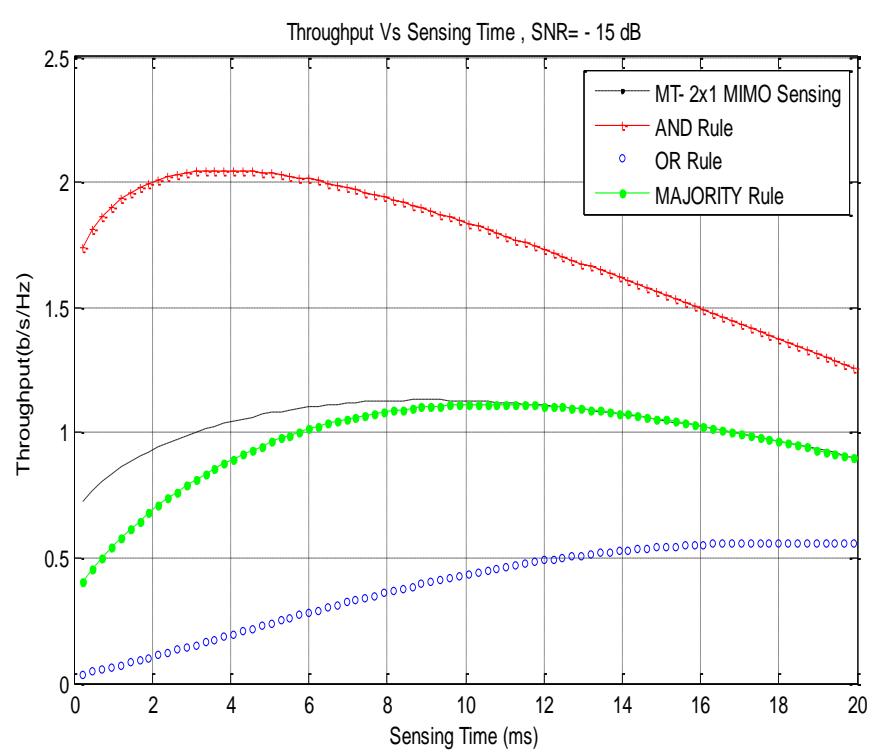

Fig.5 Throughput vs Sensing Time

(SNR=-15, K=3, MT-2x1 MIMO)

The different fusion rules of cooperative spectrum sensing are compared in case of PED 3x1 MIMO; the achievable throughput is $(0.87,1.85,0.2$, and 0.72$)$ at sensing time $(8,4$, 18 and 12) msec for (PED $3 \times 1$ MIMO, AND, OR, MAJORITY) respectively as shown in Fig.6. But the achievable throughput is $(1.25,2.2,0.75,1.25)$ at sensing time $(8,2.8,15,8)$ in case of (MT- 3x1 MIMO, AND, OR, MAJORITY) respectively as shown in Fig.7.

Achievable throughput is $(1.37,2.2,0.98,1.37)$ at sensing time $(7,3,12,7)$ in case of (PED- 2x1 MIMO, AND, OR, MAJORITY) respectively as shown in Fig.8. But the achievable throughput in case of MT-2x1 MIMO is $(1.75,2.36$, 1.46 , and 1.68) at sensing time $(3.5,1,5,2)$ for (MT- $3 \times 1$ MIMO, AND, OR, MAJORITY) respectively as shown in Fig. 9.

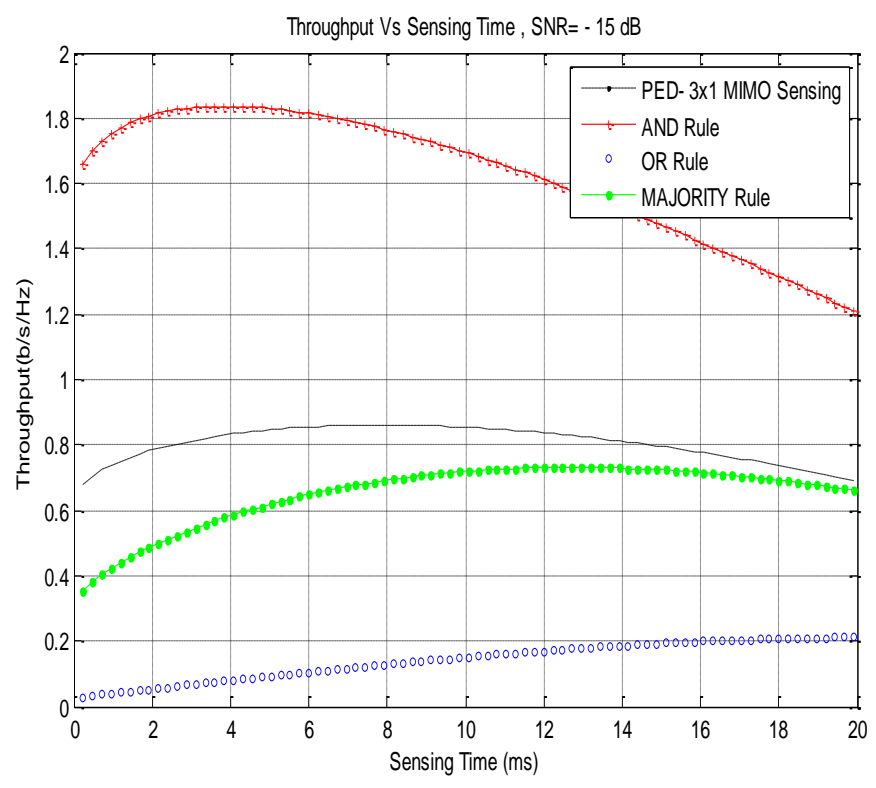

Fig.6 Throughput vs Sensing Time $(\mathrm{SNR}=-15$ PED-3x1MIMO, $\mathrm{k}=3$ )

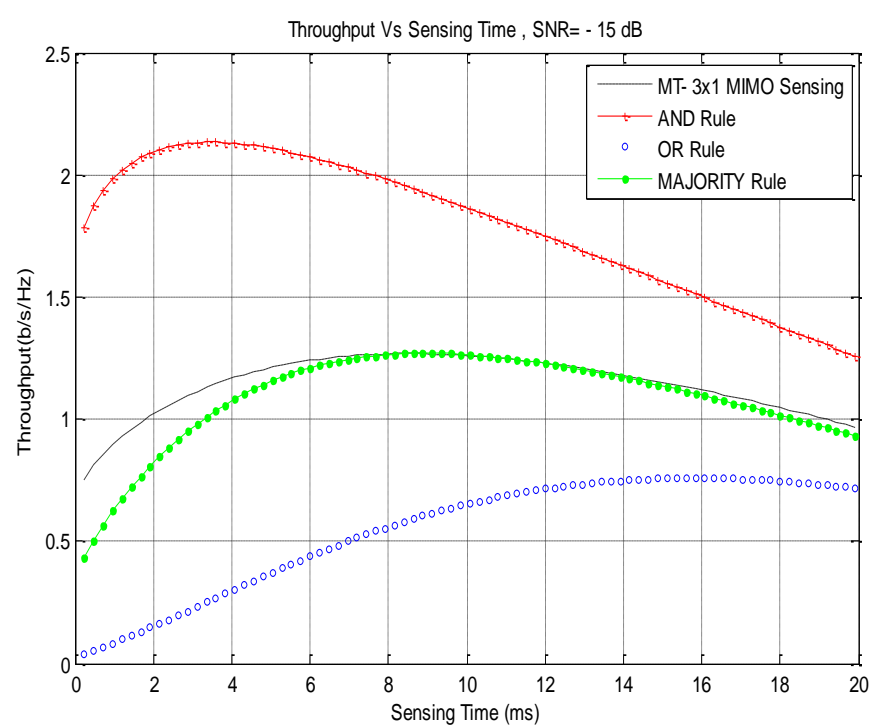

Fig.7 Throughput vs Sensing Time ( $\mathrm{SNR}=-15, \mathrm{~K}=3, \mathrm{MT}-3 \times 1 \mathrm{MIMO})$

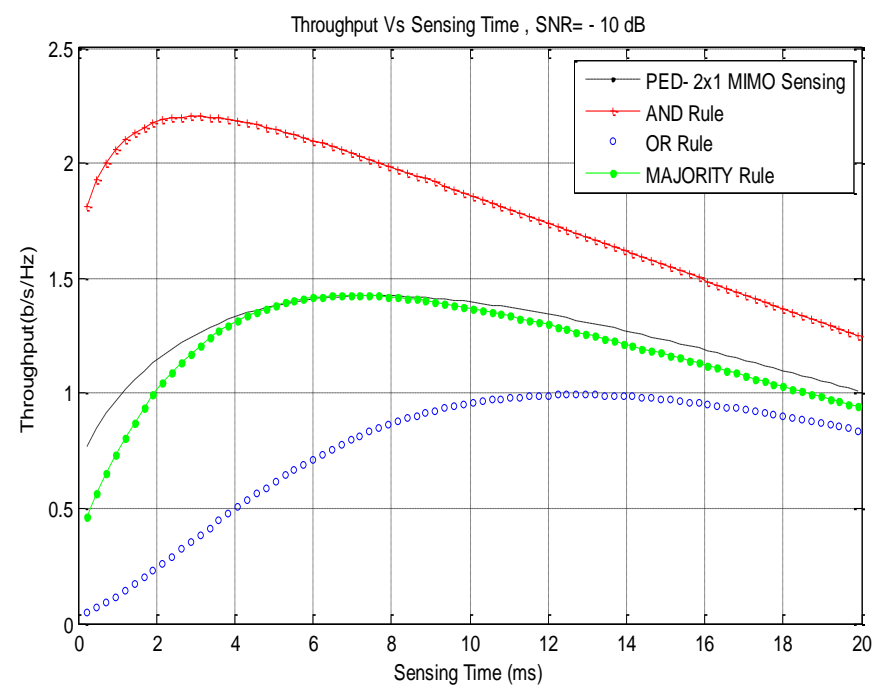

Fig.8 Throughput vs Sensing Time $(\mathrm{SNR}=-10, \mathrm{~K}=3$, PED-2x1 MIMO)

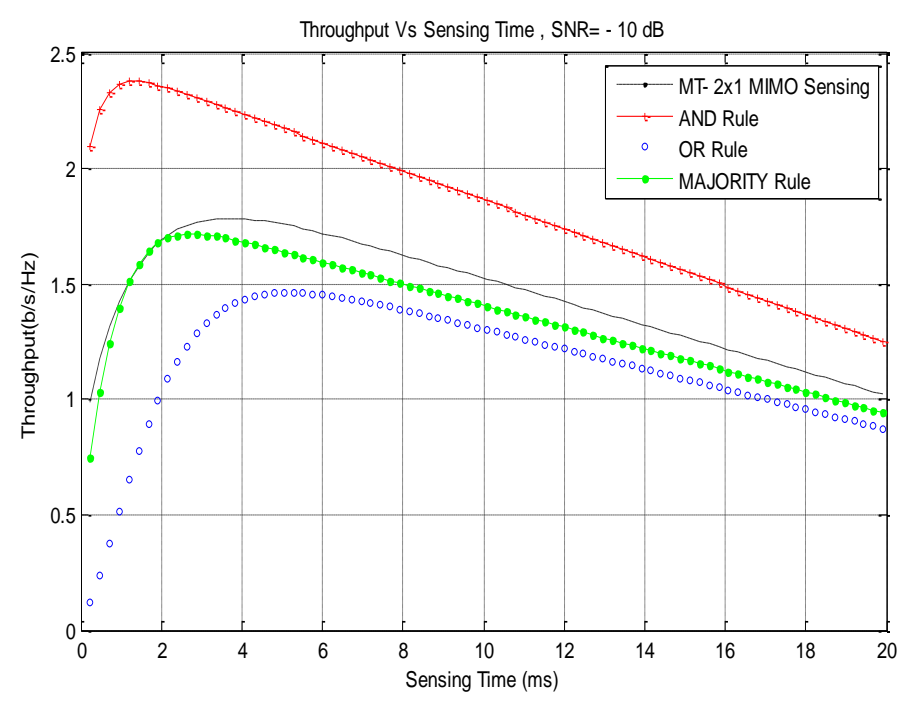

Fig.9 Throughput vs Sensing Time ( $\mathrm{SNR}=-10, \mathrm{~K}=3$, MT-2x1 MIMO) 
By increasing the number of antennas to $3 \times 1$ MIMO under conditions ( $\mathrm{SNR}=-10 \mathrm{~dB}$ and $\mathrm{K}=3$ ); the achievable throughput is $(1.57,2.27,1.2,1.57)$ at sensing time $(6,2,10,5)$ in case of (PED 3x1 MIMO, AND, OR, MAJORITY) respectively as shown in Fig.10. But in case of MT-3x1 MIMO spectrum sensing , the achievable throughput is $(1.75,2.4,1.55,1.75)$ at sensing time $(3,1,4,2)$ in case of (MT 3x1 MIMO, AND, OR, MAJORITY) respectively as show in Fig.11. All above results indicates that the MT-MIMO spectrum sensing can improve sensing capability at low sensing time with maximizing the achievable throughput in case of MT-MIMO more than PEDMIMO.

The achievable throughput can be improved in case of MTMIMO more than the PED-MIMO case by increasing number of antennas used at CR receiver; however MT-MIMI takes much sensing time. The achievable throughput is $(0.72,1.68$, $0.08,0.45)$ at sensing time $(4,2,18,9)$ for (PED 10x1 MIMO, AND, OR, MAJORITY) respectively as shown in Fig.12. But in case of MT-10x1MIMO spectrum sensing; the achievable throughput is $(0.93,1.92,0.3,0.85)$ at sensing time $(7,4,18$, 12) for (MT-10x1 MIMO, AND, OR, MAJORITY) respectively as shown in Fig.13.

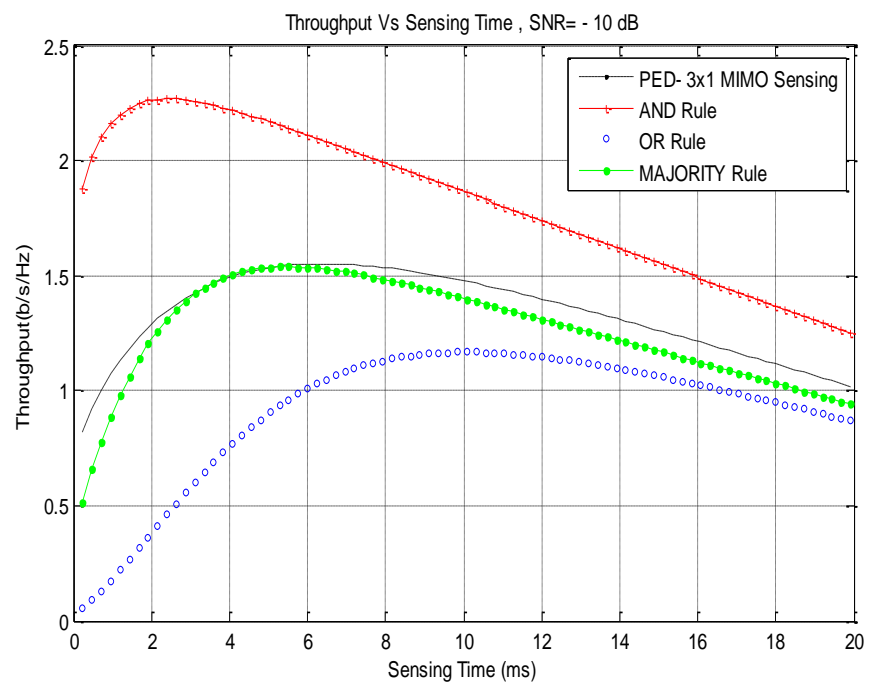

Fig.10 Throughput vs Sensing Time (SNR=-10, K=3, PED-3x1 MIMO)

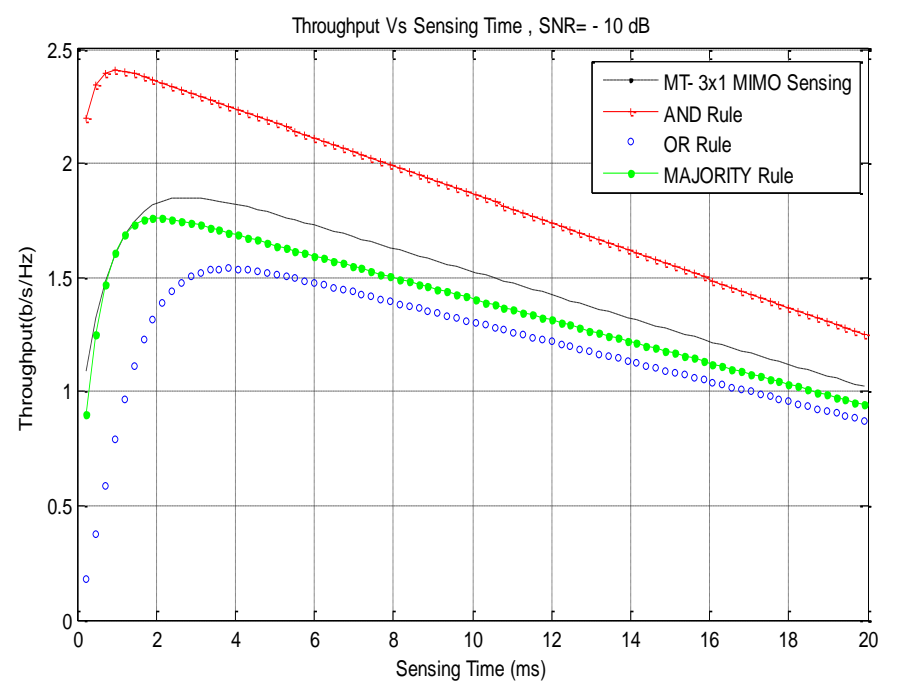

Fig.11 Throughput vs Sensing Time
$(\mathrm{SNR}=-10, \mathrm{~K}=3, \mathrm{MT}-3 \times 1 \mathrm{MIMO})$

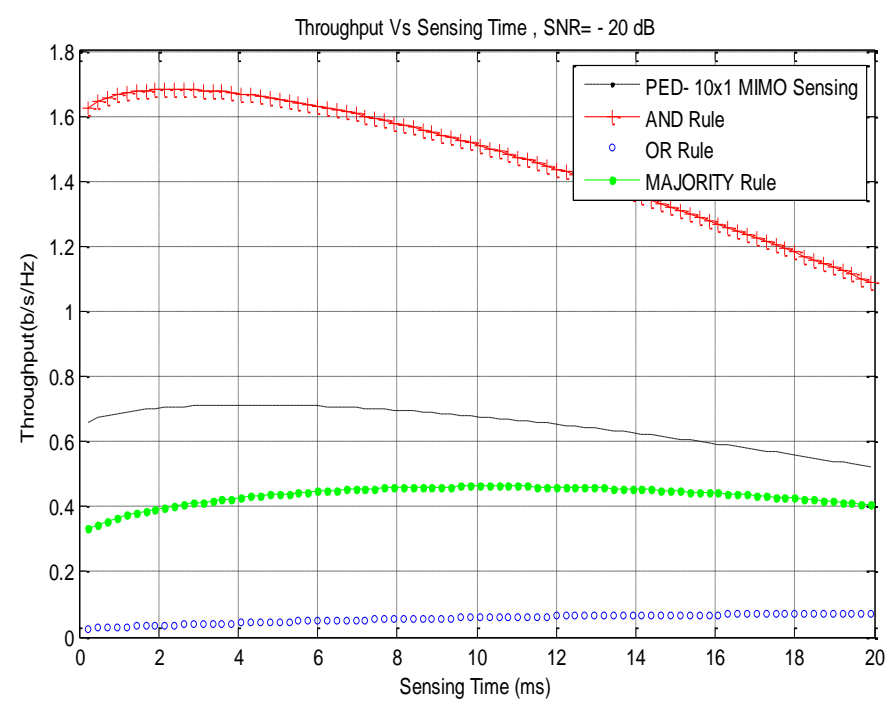

Fig.12 Throughput vs Sensing Time (SNR=-20, K=3, PED-10x1 MIMO)

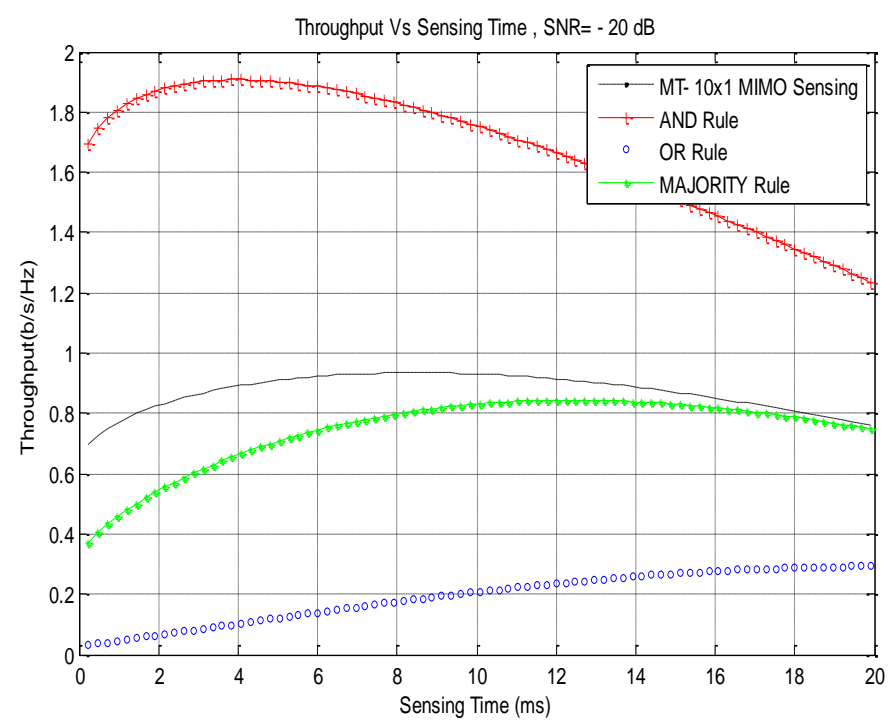

Fig.13 Throughput vs Sensing Time (SNR=-20, K=3, MT-10x1 MIMO)

\section{V.CONCLUSIONS}

In this paper, we proposed a cognitive radio system that improves the achievable throughput of secondary user by performing data transmission and spectrum sensing at the same time. We studied the average achievable throughput of the proposed cognitive radio system under single user detection and multiple user detection. The simulation results showed that the achievable throughput have been improved in case of MTMIMO compared to the PED-MIMO cognitive radio systems. In addition to the ability to solving the hidden node problem which make more protection for primary user. Also, Cooperative MT-MIMO Spectrum sensing is more efficient than PED-MIMO Cooperative spectrum sensing specially at low SNR of PU. 


\section{REFERENCES}

[1] Mohamed Ismail, Atef Ghuniem and Abdelhamid Gaafar " Performance Enhancement of Achievable Throughput in MultiTaper Spectrum Sensing" IJERT Vol. 7 Issue 04, April-2018.

[2] Force, S. "Spectrum policy task force report." Federal Communications Commission ET Docket 02, vol. 135 (2002).

[3] Mitola, Joseph, and Gerald Q. Maguire. "Cognitive radio: making software radios more personal." IEEE personal communications 6.4 (1999): 13-18.

[4] Chiang, Tsung-Wei, Jung-Mao Lin, and Hsi-Pin Ma. "Optimal detector for multitaper spectrum estimator in cognitive radios." Global Telecommunications Conference, 2009. GLOBECOM 2009. IEEE. IEEE, 2009.

[5] Haykin, Simon. "Cognitive radio: brain-empowered wireless communications." IEEE journal on selected areas in communications 23.2 (2005): 201-220.

[6] Cabric, Danijela, Shridhar Mubaraq Mishra, and Robert W. Brodersen. "Implementation issues in spectrum sensing for cognitive radios." Signals, systems and computers, 2004. Conference record of the thirty-eighth Asilomar conference on. Vol. 1. Ieee, 2004.

[7] Sun, Chunhua, Wei Zhang, and Khaled Ben Letaief. "Cooperative spectrum sensing for cognitive radios under bandwidth constraints." Wireless Communications and Networking Conference, 2007. WCNC 2007. IEEE. IEEE, 2007.

[8] Bai, Zhiquan, et al. "Cluster-based cooperative spectrum sensing for cognitive radio under bandwidth constraints." Communication Systems (ICCS), 2010 IEEE International Conference on. IEEE, 2010.

[9] Ganesan, Ghurumuruhan, and Ye Li. "Cooperative spectrum sensing in cognitive radio networks." ." New Frontiers in Dynamic Spectrum Access Networks, 2005. 2005 First IEEE International Symposium on. IEEE, 2005.

[10] Ghasemi, Amir, and Elvino S. Sousa. "Collaborative spectrum sensing for opportunistic access in fading environments." New Frontiers in Dynamic Spectrum Access Networks, 2005. DySPAN 2005. 2005 First IEEE International Symposium on. IEEE, 2005..

[11] Mishra, Shridhar Mubaraq, Anant Sahai, and Robert W. Brodersen. "Cooperative sensing among cognitive radios." Communications, 2006. ICC'06. IEEE International conference on. Vol. 4. IEEE, 2006.

[12] Axell, Erik, et al. "Spectrum sensing for cognitive radio: Stateof-the-art and recent advances." IEEE Signal Processing Magazine 29.3 (2012): 101-116.

[13] Ahmed O. Abdul Salam; Ray E. Sheriff; Saleh R.Al Araji; Kahtan Mezher; Qassim Nasir" Multi-Taper and MIMO Techniques for spectrum sensing in cognitive radio" 2015 IEEE International Conference on Electronics, Circuits, and Systems (ICECS)

[14] Alghamdi, Owayed A., and Mosa A. Abu-Rgheff. "Local MTMSVD based spectrum sensing in SIMO OFDM cognitive radio under bandwidth constraint." Cognitive Radio Oriented Wireless Networks \& Communications (CROWNCOM), 2010 Proceedings of the Fifth International Conference on. IEEE, 2010.

[15] Wang, Beibei, and KJ Ray Liu. "Advances in cognitive radio networks: A survey." IEEE Journal of selected topics in signal processing 5.1 (2011): 5-23.

[16] Papoulis, Athanasios, and S. Unnikrishna Pillai. Probability, random variables, and stochastic processes. Tata McGraw-Hill Education, 2002.

[17] Sun, Hongjian, et al. "Wideband spectrum sensing for cognitive radio networks: a survey." IEEE Wireless Communications 20.2 (2013): 74-81. 